\title{
Correction to: Puerarin alleviates burn-related procedural pain mediated by $\mathrm{P}_{2} \mathrm{X}_{3}$ receptors
}

\author{
Xin $\mathrm{Li}^{1}$ • Jun Zhang ${ }^{1} \cdot$ Yun Gao ${ }^{1}$ - Yang Yang ${ }^{2}$ - Changshui $\mathrm{Xu}^{1}$ • Guilin $\mathrm{Li}^{1}$ • Guanghua Guo ${ }^{3}$ - Shuangmei Liu ${ }^{1}$. \\ Jinyan $\mathrm{Xie}^{1} \cdot$ Shangdong Liang ${ }^{1}$
}

Accepted: 15 September 2020 / Published online: 26 September 2020

(C) Springer Nature B.V. 2020

\section{Correction to: Purinergic Signalling (2011) 7:489-497} https://doi.org/10.1007/s11302-011-9248-5

Due to the authors' carelessness, we used mistakenly PBMCs isolated from same patient in Fig. 1a for P2X3 immunoreactivity in VI: PUE-treated group on the second day (Third row middle graph) and VII: PUE-treated on the third day (Third row right- side graph).
Fig. 1 Effect of puerarin on the expression of $\mathrm{P} 2 \mathrm{X} 3$ receptors in peripheral blood mononuclear cells (PBMCs) by immunohistochemistry. a I: healthy volunteers (control); II: normal saline (NS)-treated group on the first day; III: NS-treated group on the second day; IV: NS-treated group on the third day; V: puerarin (PUE)-treated group on the first day; VI: PUE-treated group on the second day; VII: PUE-treated on the third day. (Arrows indicate the immunostaining of PBMCs; scale bars, $20 \mu \mathrm{m}$ )

Xin Li, Jun Zhang, Yun Gao, and Yang Yang are jointly first authors.

The online version of the original article can be found at https://doi.org/ 10.1007/s11302-011-9248-5

Shangdong Liang

liangsd@hotmail.com

1 Department of Physiology, Medical College of Nanchang University, Nanchang, Jiangxi 330006, People's Republic of China

2 Department of First Clinical Medicine (2 class-2007 grade), Medical College of Nanchang University, Nanchang, Jiangxi 330006, People's Republic of China

3 First Affiliated Hospital, Medical College of Nanchang University, Nanchang, Jiangxi 330006, People's Republic of China 
Original Fig. 1a

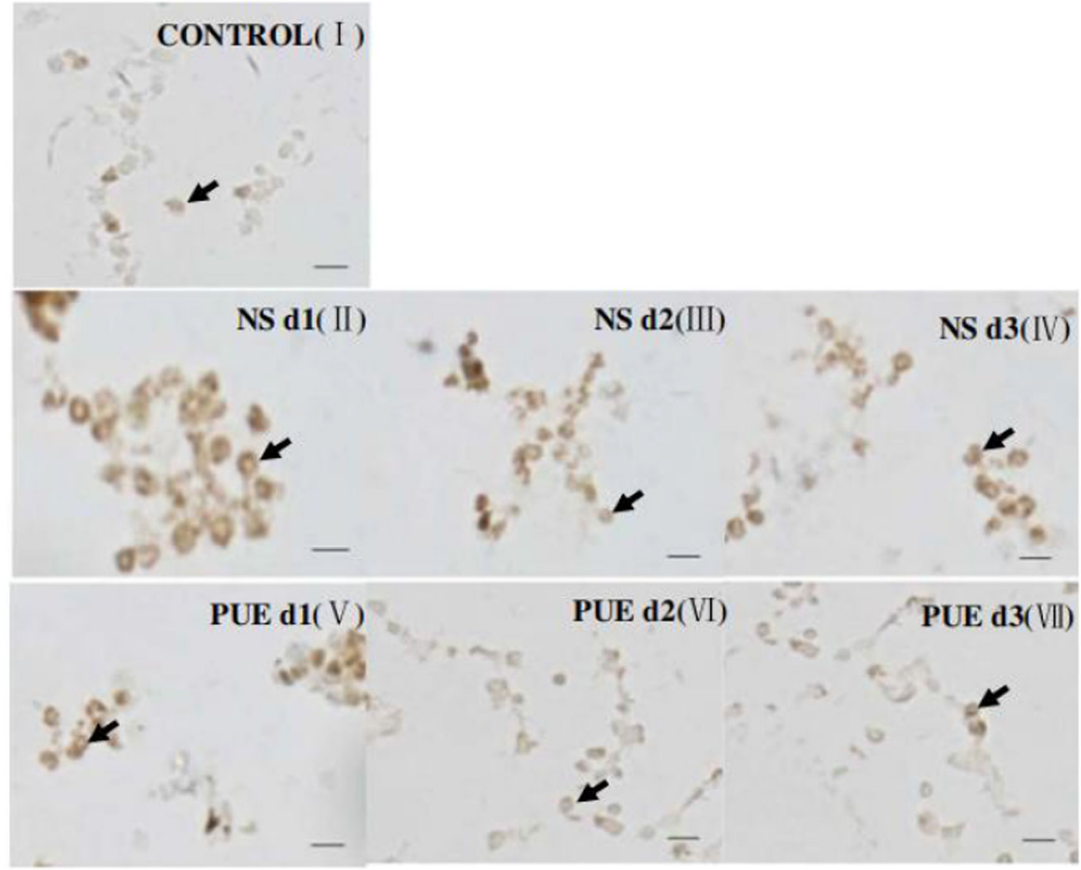

The photo of P2X3 immunoreactivity in VII: PUE-treated on the third day (Third row right- side graph) are replaced.

Corrected 1a

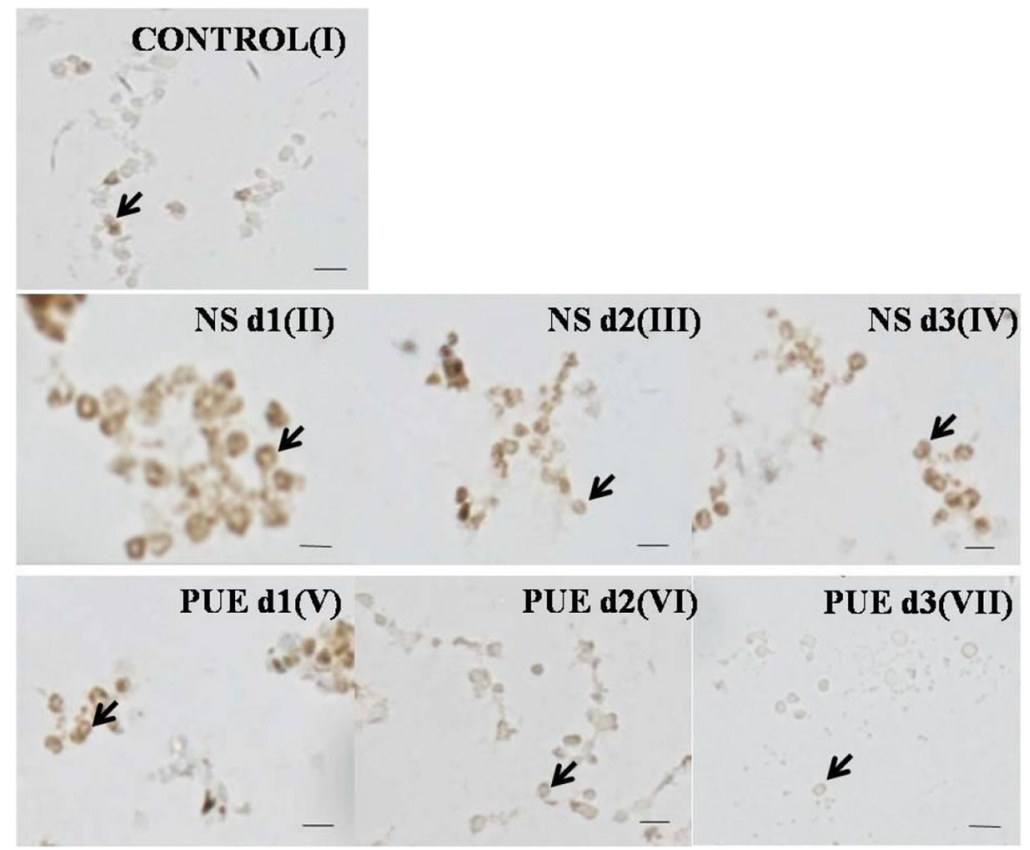

Finally, we regret the errors on our part, as we did not catch the mistake during editing, and apologize for any confusion this may cause.
Publisher's note Springer Nature remains neutral with regard to jurisdictional claims in published maps and institutional affiliations. 